Voix et Images

voixetimages

\title{
Pierre Baillargeon intime
}

\section{André Gaulin}

Volume 1, numéro 1, septembre 1975

\section{Hubert Aquin}

URI : https://id.erudit.org/iderudit/013985ar

DOI : https://doi.org/10.7202/013985ar

Aller au sommaire du numéro

\section{Éditeur(s)}

Les Presses de l'Université du Québec

\section{ISSN}

0318-9201 (imprimé)

1705-933X (numérique)

Découvrir la revue

Citer cet article

Gaulin, A. (1975). Pierre Baillargeon intime. Voix et Images, 1(1), 57-71.

https://doi.org/10.7202/013985ar d'utilisation que vous pouvez consulter en ligne.

https://apropos.erudit.org/fr/usagers/politique-dutilisation/ 


\section{Pierre Baillargeon intime}

II y avait plusieurs Pierre Baillargeon, les uns connus, les autres inconnus, tous cependant compacts, rugueux, et qui, réunis, formaient une masse opaque, quasi impénétrable, difficilement accessible. Quelle étrange personnalité que la sienne! d'une étrangeté impassible, fixe, immuable, telle à quinze ans qu'à cinquante. Que fut-elle? Dieu le sait mieux que moi, son camarade de collège et d'université, maintes fois son compagnon de voyage, longtemps son voisin de chambre, et, à Paris, son guide. Il s'était retranché en lui-même comme en un donjon. Comment entrer?

C'est Paul Toupin qui parle ainsi dans le portrait qu'il a fait de Pierre Baillargeon ${ }^{1}$. Je fus étonné de lire ce texte sinon hostile du moins froid et peu sympathique sous la plume de celui qui devait connaître Baillargeon dès le collège Brébeuf. N'est-ce pas le même Toupin qui rappelle fort à propos, dans le Nouveau Journal, des souvenirs de cette époque: "Au collège que j'avais fréquenté ici, notre classe était divisée en claudéliens et valéryens. Pierre Baillargeon et moi étions valéryens. II y avait également des mauriaciens, des bernanosiens et je me demande s'il faut voir un signe dans ceci que de nous tous, seuls Baillargeon et moi-même sommes devenus écrivains ${ }^{2}$." Bref, cet article un peu mesquin étonnait de la part de celui que Baillargeon considère dans son journal personnel un peu comme un ami. Que fallait-il donc à monsieur Toupin pour mieux connaître l'auteur des Médisances? La lecture de l'œuvre ne livre-t-elle pas Baillargeon tout entier, le moraliste idéaliste, l'essayiste exigeant un environnement culturel fondé sur la recherche de l'intelligence, l'homme pas toujours sûr de lui-même, caché derrière ses phrases lapidaires, l'écrivain épris du goût français de la clarté et un peu déçu d'un milieu culturel médiocre? Serait-ce donc que les écrivains d'ici ne se lisent pas entre eux et Pierre Baillargeon avait-il vraiment été lu par son commensal Paul Toupin? Citant Baillargeon, je dirais que «tant de silence est une chose effrayante ${ }^{3}$ ".

Une voix s'éleva pour défendre Pierre Baillargeon, et qui était celle d'une Française, Madeleine Ducrocq-Poirier ${ }^{4}$. Madame Poirier qui n'a pas eu comme Toupin le bonheur de fréquenter Baillargeon trouve abon- 
damment, dans l'œuvre de ce dernier, le contre-témoignage de monsieur Toupin. Et moi aussi, je dois le dire. Comment croire à la froideur et à la carapace de cet homme qui éclate de tendresse presque, à la fin des Médisances de Claude Perrin? Comment croire à l'opacité de celui qui a donné au Québec le si fragile et touchant Philippe Boureil de la Neige et le feu? Est-il vraiment impénétrable le libraire Claude Perrin de Commerce qui est essentiellement un homme de conversation et un homme d'esprit comme Baillargeon le fut? J'espère encore qu'il sera bientôt donné à nos contemporains le plaisir de lire Autour d'un gros bonhomme, roman inédit, où la vision du monde de Baillargeon est celle du philosophe soucieux du bonheur des hommes.

Je ne connais pas monsieur Toupin autrement que pour l'avoir lu et je l'imagine aussi tel un être mangé par l'écriture, malhabile à vivre comme ses personnages, mystérieusement hanté par le souvenir qui est tout autant en avant de lui qu'en arrière. Dévoré par l'écriture, oui, et consumant sa vie à cela comme le héros de Souvenirs pour demain 5 ou celui de Mon mal vient de plus loin 6 . La fréquentation d'une œuvre aussi autobiographique que celle de Pierre Baillargeon ne peut nous permettre de le méconnaître. A titre d'exemple, je propose ce petit extrait significatif des Médisances de Claude Perrin:

La vraie fin de l'homme, ce n'est pas la mort, c'est sa perfection. Celle-ci peut être atteinte ici-bas. Le plus souvent, elle se projette au delà de l'existence.

A bien des hommes il a manqué quelques années pour s'accomplir. [Baillargeon mourut à cinquante et un ans.] Si l'on veut les connaître à fond, besoin est d'imaginer achevées leurs œuvres, sublimes leurs vertus, réalisé leur idéal. Pour tracer le cercle, on prolonge la courbe.

Au contraire, ne tenir compte que de ce qu'ils ont fait et dû, par conséquent, abandonner, c'est ordinairement les dénigrer.

D'un être cher à soi, on n'a, d'ailleurs, jamais fini d'approfondir la connaissance. Cette recherche passionnée, cette étude, science et goût tout à la fois, est l'essence de l'amitié.

Connu, jugé, je ne suis plus qu'une connaissance.

Si l'on me juge de mon vivant, on me diminue, on m'extermine presque. C'est aussi que l'on se désintéresse de moi. TOUT JUGEMENT EST UNE ÉPITAPHE?.

Comment parler de la froideur de cet homme humain qui écrit, par exemple: "Le soir, quand il fait beau, et que les automobiles trop nombreuses n'en gâtent pas l'exercice, j'aime faire une promenade à pied dans le quartier. Je rêve, je regarde les arbres. J'admire le peuplier d'Italie dont le faîte agité par la brise semble peindre à grands coups de brosse un coin de ciel. Je vais donc, le nez en l'air ${ }^{8}$." II y a ici la présence de l'arbre qui domine dès 1940, l'cuure de Pierre Baillargeon. C'est l'homme enraciné, intérieur, et qui bien que fixé en terre, voyage et reste ouvert sur le monde. Celui qui termine Le scandale est nécessaire 
par sa devise, image d'un arbre dont la légende serait: Enfonce-toi et domine ${ }^{9}$ revient au saule dont il parlait déjà dans Amérique française en $1941^{10}$. Le Père Robert Bernier, un ami fidèle de Baillargeon, a repris ce poème dans sa préface de Choix ${ }^{11}$. Nous savons, par le journal personnel, que le poète avait complètement retouché le texte ${ }^{12}$. Nous aurions là à la rigueur la clé de l'œuvre, puisque ce saule "vagabonde", "captif au bord de l'eau». L'écrivain qui s'est bien regardé ne l'a pas fait comme Narcisse pour se trouver beau mais à la manière de Montaigne qui se disait microcosme du monde. S'il est vrai que l'écrivain n'a pas tellement changé de vingt ans à cinquante, ce n'est pas pour s'être répété ou redit mais plutôt approfondi. Le Claude Perrin des Médisances ne disait-il pas justement:

De mon enfance, je ne dirai que peu de chose, guère davantage. Je sais bien qu'on ne change pas. Que l'on parle de sa jeunesse ou de son âge mûr, au fond, on travaille au même portrait. On grandit avec ses défauts et ses qualités. L'ombre croît en même temps que l'arbre. C'est l'erreur des éducateurs de vouloir nous changer ${ }^{13}$.

L'écrivain Baillargeon fut un introverti sans doute, à un point tel qu'il avait l'air distrait ou distant, mais un introverti grégaire. Ce qui lui a valu l'expérience de l'approfondissement de la solitude. Le Perrin de Commerce n'affirme-t-il pas que «la solitude est le principal avantage que procure la société ${ }^{14}$ "? Le même Perrin sait très bien aussi que "tout solitaire est fondateur d'une société nouvelle ${ }^{15}$. Si Perrin est égocentrique, il est autant tous les hommes. Un passage magnifique de Commerce serait à citer:

Celui qui s'étudie à l'écart, et qui se goûte seul, ne s'éloigne guère des autres. En lui-même il découvre toutes les personnes. Daniel Defoe a illustré cela: Robinson Crusoé s'entoure d'une foule imaginaire dont il joue lui-même les divers rôles: tour à tour potier, maçon, chasseur, cultivateur, soldat, voire littérateur, car il finit par écrire son propre journal ${ }^{16}$.

Pierre Baillargeon n'avait donc rien d'un misanthrope. Mais il est un écrivain tel, que besoin est de fréquenter son œuvre. A le lire trop rapidement, ou à ne pas le relire, on peut être porté à méconnaître l'auteur. Ainsi, il est frappant de constater que celui qui devait faire un éloge de Pierre Baillargeon, le professeur Jean Ménard ${ }^{17}$, alors qu'il a lu les propos de Paul Toupin, se met à douter de l'homme qui écrivit les Médisances ${ }^{18}$. Bien sûr, Jean Ménard ne répudie pas plus Baillargeon que Toupin, je pense, n'a voulu desservir la mémoire de son ami, mais dans l'un et l'autre cas, voilà que s'élève le doute. Dans le cas de monsieur Toupin, il y a comme l'oubli de ce que l'écrivain a dit, une absence de bienveillance qui étonne surtout de la part d'un ami.

Je sais bien que Pierre Baillargeon ne fut pas un écrivain facile mais, justement, besoin est de l'expliquer, de le comprendre. Si l'auteur fut parfois dur dans ses jugements sur ses compatriotes, c'est qu'il les 
aimait. II souffrait d'un pays souvent francophobe, francophobie dont il rendait partiellement le clergé responsable, il éprouvait la gêne de celui qui veut consacrer sa vie à l'écriture et que le milieu réduit à dire des banalités pour être bien reçu, que l'environnement culturel prive de lecteurs. Ses œuvres d'avant 1948 furent reçues par des critiques souvent plus moralisateurs qu'intelligents; à son retour au pays en 1960 , on ne le connaissait pratiquement plus dans la jeune génération et sa conception classique de l'écriture faisait qu'il ne comprenait pas toujours le succès de la poésie ou du roman québécois contemporains. De plus, Baillargeon s'était réfugié dans un genre difficile, celui de la maxime, qui le manifestait tel qu'il était, une sorte d'anti-romancier, un essayiste qui ne visait pas le développement d'une pensée ou d'une réflexion cohérente mais plutôt un essayiste moraliste qui tendait vers une vision du monde et de l'homme qui se composerait comme une mosaïque. Être l'auteur d'un seul livre, voire d'une seule maxime. Son œuvre tendait au silence. Le Perrin des Médisances ne disait-il pas qu'écrire, c'est choisir ${ }^{19}$ ? Pour lui, «une œuvre littéraire doit être concise. Les mots inutiles sont des moments perdus. [...] L'on doit se borner à l'essentiel, c'est-à-dire, à soi-même. L'essentiel ici tient dans chaque instant d'une longue vie. II pourrait aussi tenir en une phrase. Si l'on en écrit généralement plusieurs, c'est par crainte de manquer la seule bonne ${ }^{20}$ ». Baillargeon, d'ailleurs, n'a-t-il pas fait dire à Claude Perrin, dès 1945 , - en partie du moins - pourquoi il ne fut pas $l^{21}$. Et dans Commerce, il se décrit très bien dans sa mort, entouré de quelques amis fidèles et s'en retournant au silence. Ce sonnet qui est intitulé "la Fin d'un homme de lettres" reste simplement émouvant:

Quelques amis viendront peut-être

Me chercher, riant ou pleurant;

Et je m'en irai lentement

Vers l'église où m'attend le prêtre.

Les voisins, tous indifférents

$\hat{A}$ la fin d'un homme de lettres,

Ne se montreront aux fenêtres;

Je passerai nul comme avant.

Et quant à mon petit cortège,

On croira que c'est des passants.

lis loueront tout bas mon talent;

Mais leurs mots feutrés par la neige

Seront pour moi, si Dieu le veut,

Meilleurs que le plus tendre aveu ${ }^{22}$.

Pierre Baillargeon eut-il raison de viser ainsi au dépouillement de l'écriture? Bien des critiques devaient lui reprocher sa sécheresse ou ce qu'il jugeait comme tel. II faudra, sans lire entre les lignes, attendre la parution posthume de Choix pour découvrir un Pierre Baillargeon plus concret, moins distant, encore que ceux qui avaient lu le journaliste de la Patrie, du Petit Journal connaissaient déjà ce côté plus chaud de l'écrivain. Mais c'est peut-être dans son journal, encore inédit, que les contemporains découvriront le mieux la simplicité et la grande humanité de l'essayiste montréalais. Ils y rencontreront un homme éminemment attaché 
à sa patrie, amoureux de la France, attentif à la vie politique, sociale et culturelle qui l'entoure, vivant d'amour et d'écriture. Aussi voudrais-je brièvement compléter ce témoignage sur Pierre Baillargeon en livrant quelques réflexions de son journal. Auparavant, toutefois, il y aurait un intérêt historique à reprendre ici un portrait que faisait de Baillargeon l'écrivain Jacques Ferron en 1948. Ferron a toujours admiré Baillargeon: il appréciait sûrement en lui cette sûreté de l'écriture puisque tous les deux sont des écrivains conservateurs, ce que Baillargeon aurait défini par classiques ${ }^{23}$. Ce portrait de Ferron envoyé par le médecin au journaliste de la Patrie manifeste un lecteur attentif comme Ferron sait si bien l'être de ses collègues en écriture. De plus, le croquis livre un Baillargeon pas tellement soucieux de l'homme pauvre, de la vie ambiante: en quoi, le journal démontre que Baillargeon était non seulement réservé, mais profondément valéryen par son attachement aux idées.

Quand j'aperçois Pierre Baillargeon dans la rue, je ne l'aborde pas, car ne cessant pas de lui écrire, si je l'abordais par surcroît, je ne tarderais pas, fâcheur, à le fấcher; comme j'ignore de quelle façon il le fait, je redoute cet inconnu et je le suis de loin.

Je le suis, craignant pour lui quelque accident: le poète le plus agile est toujours menacé dans la rue, car faisant un pas par hémistiche, il est dangereux que la fin de la strophe ne l'arrête au milieu de la chaussée. Je le surveille donc en le suivant.

A sa sortie de la Patrie, ayant expiré jusqu'à sa dernière molécule l'atmosphère du journalisme, et ne pouvant demeurer sans respiration, Baillargeon inspira l'air froid de la ville; puis, il regarda le ciel entre les fils téléphoniques et les maisons hideuses: aucune étoile insolite n'y brillait; il n'eut pas à devenir roi-mage, et il se contenta, en respectant la césure, - car il est classique comme on ne l'est plus depuis Hugo, - d'ajouter un pas à l'autre et d'aller ainsi au gré de son poème intérieur, lequel, en l'occurrence ressemblant fort à son appétit, le mena droit à l'Hôtel d'Italie.

II porte un feutre brun, rabattu, sans autre prétention que de coiffer son homme; une barbe noire, forte et qu'on voit poindre à la moindre fatigue; des yeux de même couleur, qui sont beaux et qui s'ignorent à bon escient car le souci de se connaître les entraînerait à loucherie; des yeux francs qui sont d'ailleurs ennuyés de voir tous deux la même chose - un seul suffirait à son regard perçant, mais le poète est trop connu pour pouvoir cligner de l'œil.

Avec tous ces attributs et d'autres encore dont le moins apparent n'est pas son embonpoint, il pénètre dans l'Hôtel, enlève son chapeau et déclare à l'assemblée qu'il est chauve; depuis Verlaine c'est un détail qui rajeunit, et Baillargeon ne cache pas la satisfaction enfantine qu'il éprouve à la pensée que le macaroni sera délicieux.

II n'y a pas de doute que ce maître du style, qui a parfois une petite tendance à jouer les maîtres d'école, qui prétend que sans le subjonctif imparfait il n'y a pas de salut, soit l'homme le plus ingénu et le plus spontané du monde. C'est dans ce contraste, je crois, que se trouve le charme de sa personne.

Comme Boileau et Baudelaire, il ignore la campagne, et la nature pour lui est un jardin de ville; il ignore les animaux, le doux naseau 
du cheval, et le corbeau, séminariste débauché devenu intendant, qui surveille les préparatifs à la venue du printemps. Les yeux clairs et le regard franc du peuple lui sont inconnus; il a des pauvres gens une idée pitoyable; ceux-ci d'ailleurs sont rares sous sa plume, et dans Commerce un seul paragraphe leur est accordé à l'occasion d'un vidangeur. (Mais il faut convenir que ce paragraphe direct est extraordinaire.)

Pierre Baillargeon, par sa formation et son milieu, est en marge de la vie $^{24}$. Il court le risque de tout classque d'être pédant. Mais il a pour l'en préserver cette enfance dont je parlais, cette flamme pure qu'il y avait dans les yeux de Shelley et de Mallarmé, qui, comme lui, vécurent en réclusion et n'eurent pour s'évader qu'une feuille blanche devant eux, une feuille sur laquelle une nouvelle captivité a commencé, celle-là éternelle, qui nous a transmis le reflet de leurs yeux.

Or donc, je suis Pierre Baillargeon dans la rue, et je ne puis me lasser de sa maladresse absurde et délicieuse; comme je ne me serais lassé de surveiller Mallarmé sur la Seine. $Y$ a-t-il rien de plus extraordinaire, de plus irréel, de plus enfantin que ce vieux professeur d'anglais dans une chaloupe? Même sans doute qu'il est un corsaire redoutable, que son Trois-mâts secoué par la tempête entrera demain dans une baie lumineuse où précédée par l'odeur des verts tamariniers, sur une nacelle, la reine de Saba viendra à sa rencontre.

Les poètes sont des êtres absurdes et leurs poèmes sont de même car il n'est rien de plus insensé que leur charme, et rien néanmoins de plus sensible.

Et je regarde Pierre Baillargeon qui s'avance sur le trottoir, obéissant aux nécessités de son poeme, qui ne sont pas toujours celles de la circulation urbaine. Je soupire d'aise quand je le vois disparaître dans l'Hôtel d'Italie.

Cette fable amicale étant achevée contre mon vouloir, par cette disparition mystérieuse, je vous demanderai, non certes de me parler du repas que vous fîtes dans le mystère, mais de m'accorder, mon cher Pierre, une chose qui me plairait beaucoup: celle d'écrire un Bulletin du mois (genre NRF) dans votre revue. Vous me ferez si grand plaisir que pour vous le rendre, j'y serai méchant pour ces communistes de malheur qui nous arrivent avec une religion nouvelle contre toutes les lois du classicisme, et à un âge de votre vie où la sécurité sociale est nécessaire à votre gloire.

Je vous serre la main, Jacques Ferron 25

Pierre Baillargeon a laissé de nombreux cahiers de notes et un journal écrit par périodes de 1940 à 1967, année de sa mort. Le tout a été conservé tant bien que mal à travers ses nombreux déménagements à Montréal, en Normandie, à Vézelay, à Paris. Un auteur montréalais m'avait laissé croire que Pierre Baillargeon avait «vaniteusement "ramassé tout ce que l'on avait écrit sur lui: tel n'est pas le cas puisque le journal 
lui-même n'a été sauvé (et peut-être partiellement) que par la curiosité et l'intérêt de la famille. Voici donc quelques extraits significatifs tirés de cette somme volumineuse de petits cahiers. C'est ainsi qu'en juillet 1948, Baillargeon lisant Montaigne écrit:

«J'ai une apparence favorable sur le simple crédit de ma présence et de mon air, des personnes qui n'avaient aucune connaissance de moi s'y sont grandement fiées... et en ai tiré en pays étranger des faveurs singulieres et rares." (Montaigne)

Je pourrais presque dire le contraire à mon sujet. J'ai l'air moqueur, encore maintenant si je souris, bien que je n'ai jamais été plus qu'enjoué, et que j'aie toujours répugné à rire d'autrui, soit d'un défaut physique ou d'une imbécilité; je n'ai jamais fait d'esprit qu'aux dépens des fats et des méchants, de tous ceux qu'ils jugent à tort ou à raison, plus souvent à tort, plus sots qu'eux. Au college, il suffisait que le maître me regardât une fois, une première fois, pour que je comprisse à son air, qu'entre nous deux, c'était la guerre. Dossier? Mes condisciples. Les autres de mon àge me reprochaient d'être différents d'eux. «ll veut être original." "ll dort tout le temps, Baillargeon" disait encore le professeur Bisson s'il me voyait les yeux fermés quand en fait je réfléchissais. Distant quand je n'étais que distrait. Prétentieux quand je n'étais que consciencieux, ambitieux dans les choses désintéressées, fendant quand simplement franc, direct, sûr de ce que j'affirmais: je n'affirmais rien dont je ne fusse certain; pince-sans-rire... méprisant quand critique, froid quand pudique à l'extrême, dur quand trop sensible et recroquevillé, blessé. La peau la plus tendre est souvent rugueuse à force de croûtes, de cicatrices...

Tête forte, mais visage petit, cou gros et court mais menton délicat, poitrine très grande, jambes fines, petites mains, épaules tombantes...

Le 18 avril 1964, il ajoute:

Moi qui ai passé pour si sec, si méchant, si malveillant, je n'ai vécu que pour l'amour. Qu'est-ce que l'amour? Une obsession, une idée fixe. J'en ai connu surtout l'obsession, la déception, la passion, et rarement la joie.

II complète cette réflexion sur l'amour dès 1945 !

Ce qu'il y a d'irrationnel, donc d'injuste, dans l'amour me le rend toujours suspect. Tu m'aimes, mais pour en jouir, je voudrais savoir... J'enrage que nous ne soyions que des êtres humains! Que de chaînes, que d'illusions, que de mirages! (10 septembre)

L'écrivain souffre donc de l'image qu'il projette:

Et puis ma maudite réserve, ma maudite pudeur, qui fait que je garde pour moi mes meilleurs sentiments, mon affection, montrant tout le contraire pour les mieux enfouir en moi. (18 janvier 1964)

L'homme Baillargeon fut généreux et ouvert à la misère des autres, attaché aux siens et essentiellement fidèle. II savait que l'amour donne toute la dimension d'une vie et c'est pourquoi il écrit le premier janvier 1964: “L'amour seul peut ôter à la mort son horreur.»

Sa vision du monde est influencée par l'amour. Ainsi ce texte du 27 juillet 1960, alors qu'il vient de rentrer d'un séjour de douze ans en Europe: 
Le Canada est un pays mort. Montréal est une ville sans âme en expansion rapide comme une bombe éclate. On y est terriblement mort. C'est effrayant. Tous ces corps qui s'agitent doivent haïr ce qui ici est encore vivant. On pense toujours à gagner de l'argent pour pouvoir en dépenser plus, plus vite. L'argent, cela a fini par remplacer l'âme.

Mais sa vision du monde est tout autant influencée par l'esprit. Chez Baillargeon, on ne peut séparer l'un de l'autre. Qui mieux que Baillargeon ne l'a d'ailleurs dit; un jour que Michel Garneau (de Radio-Canada) lui demandait s'il voulait que son ceuvre se fasse par l'esprit ou par le cceur, l'auteur répondit à l'animateur de " la Revue des arts et des lettres": "Le cceur vous répond: par l'esprit." (Journal du 17 janvier 1966) On peut alors comprendre que Baillargeon ait souffert du climat culturel du Québec autant dans son expression intellectuelle que spirituelle:

Les trois ordres d'après Pascal: le corps, l'esprit et le cœur. II faut dire que sans un compte de banque, il n'y a pas de vie intellectuelle possible; pas de liberté dans la misère. Et, ce qui est plus important, là où il y a peu d'intellectualité, il y a aussi peu de spiritualité. Les saints bêtes ne vaudraient guère mieux que les tristes. Nous en savons quelque chose. Trop longtemps, au Canada, on a cru que le mépris des biens matériels et même de l'intelligence permettait d'accéder immédiatement à l'ordre supérieur de la charité. C'est pourquoi nous voyons encore aujourd'hui tant de pauvres, si peu de véritables intellectuels parmi notre fausse élite et encore moins de saints influents. Corps malades, esprits débiles, cœurs durs. (14 avril 1963)

Cette façon de voir amènera souvent Baillargeon à réfléchir sur la pauvreté. L'auteur que lit Ferron n'en a sans doute pas parlé mais ce n'est pas parce que I'homme n'y avait pas réfléchi:

L'employé n'a donc aucun droit: il doit tout vendre pour un petit salaire. (30 août 1945)

Le pauvre n'a pas de santé, parce qu'il n'a pas assez d'argent pour se nourrir bien; il n'a pas d'instruction, parce qu'il n'a pas assez d'argent pour défrayer ses cours; enfin, il n'hérite pas, parce que son père et son grand-père étaient des pauvres comme lui. Tandis que le riche, lui, a tout: santé, instruction, fortune. S'il vient à perdre la santé ou la fortune, il lui reste soit l'instruction et la santé, ou la fortune et l'instruction, c'est-à-dire assez pour recouvrer l'une ou l'autre. II est destiné à demeurer riche comme l'autre est condamné à rester pauvre. Des considérations de ce genre me hantent depuis une semaine. (20 juillet 1946)

Les étrangers, ce sont d'abord les pauvres, les pauvres de tous les pays! Ils sont étrangers au monde moderne. Ce sont des êtres intermédiaires entre les animaux domestiques et les gens qui jouissent de la civilisation. (11 novembre 1960)

Baillargeon réfléchit ainsi sur la condition humaine de la plupart des hommes qui s'agitent:

Ce qui dure plaît au cœur de l'homme. Il aime bâtir solide, retenir une femme, élever une famille, vivre avec les hommes comme lui, dans une cité... II considère comme perdue une vie livrée aux plaisirs qui dissipe une fortune, ne produit pas d'enfants, etc. Produire, 
entasser, constituer une réserve, se créer du loisir, mais employer encore ce loisir pour augmenter sa réserve, son capital, son potentiel, son avoir et son pouvoir... Ceux qui jouissent de la vie, qui dépensent leurs réserves, qui veulent vivre, ne sont tolérés que parce qu'ils facilitent aux autres, aux gens sérieux, la tâche de s'enrichir à leurs dépens... Ils travaillent encore mais pour les autres... Les autres travaillent pour leurs enfants, qui travailleront pour les leurs, et ainsi de suite. Ils ont en fait besoin d'avoir quelque chose à faire et se dégoûtent de la vie dès qu'ils prennent leur retraite. L'homme ne sait pas rien faire. Il s'agite du commencement à la fin. Même quand il jouit de la vie, il a l'air de travailler. Leur vie est absurde depuis qu'ils luttent toutes les heures pour vivre et ne vivent jamais ou s'ennuient à mort quand il n'ont rien d'autre à faire que de vivre, ce qui d'ailleurs ne se produit jamais que dans l'extrême vieillesse ou en prison peut-être. L'absurdité de leur existence étant intolérable, ils ont inventé une récompense dans une autre vie. Mais ils ne pourraient pas souffrir maintenant, sur terre, l'idéal de félicité qu'ils se promettent au ciel s'il venait à se réaliser un jour seulement. Ainsi toutes les peines, ils se les imposent pour un bonheur futur qui serait plus intolérable que toutes leurs peines ensemble: ce serait un repos sans fin... un bonheur sans fin et monotone, etc. La peine de Sisyphe aurait pu être augmentée: c'est qu'il eût enfin réussi à monter la pierre et qu'il n'eû́t plus su que faire ensuite. (13 août 1948)

On le voit, l'écrivain ne cède pas aux modes. II ne refuse pas non plus le progrès, mais il le passe au crible. Ainsi, par exemple, lorsqu'il parle de la vitesse:

L'espace est à réduire, le temps est à gagner. La conquête de l'espace ne vaut que si elle permet celle du temps. II ne s'agit pas d'aller de plus en plus vite, mais de vivre de plus en plus. La vitesse ne doit être que le moyen d'employer au mieux son temps. A toute accélération de vitesse doit correspondre une augmentation de loisir (...) Les transports modernes ont changé notre corps en projectile qui tend à rapprocher le projet et sa réalisation, à combler l'intervalle qui sépare l'intention de son but. (2 juillet 1956)

$\mathrm{Ce}$ qu'on achète le plus cher, c'est la vitesse. Plus elle est grande, plus elle coûte cher. Et on sait de moins en moins quoi faire d'elle. Elle nous ligote toujours plus solidement, à la fin on n'ose plus respirer tant on va vite, on en perd le souffle. La vitesse, à quoi ça sert? Pour aller où? Mais si vous avalisez le chemin, il n'y a plus de voyage, on est tout de suite arrivé, ce n'est pas drôle (...) Entre un lieu et un autre, il n'y a plus qu'un siège. On s'élève dans les nuages, on descend, nous voilà arrivés. Voyageurs à vapeur. Un long voyage, c'est un peu de vapeur, un nuage,... La vitesse, on en jouit assis au milieu des choses qui foutent le camp. J'aime mieux marcher au milieu des choses qui restent bien tranquilles. (19 août 1956)

L'auteur montréalais est profondément humaniste. II écrit qu'être humain "c'est être tout plein des autres. - Être humain, c'est enfermer en soi l'humanité entière». (14 juillet 1955) Cela ne l'empêche pas avec sa vision un peu classique du monde de sentir profondément comme un moderne. Ainsi, il écrit:

Je regarde maintenant les devantures des vitrines, les montres des magasins de chemises. Je me dis que ce n'est peut-être pas du 
temps perdu, qu'il y a là peut-être une forme de culture de l'esprit. (15 février 1958)

Commencer une pièce ainsi: plusieurs personnages en scène qui ne disent rien, l'un d'eux s'écrie enfin: on ne peut rester plus longtemps ensemble! Et tous, l'un après l'autre, en silence, quittent la scène. C'est la fin de la scène première. (17 mars 1955).

Bien des contemporains de Baillargeon l'auront vu comme un apolitique. Pourtant, plusieurs réflexions du journal, après 1960, manifestent un Baillargeon inquiet et attentif.

La séparation de la province de Québec, je la vois surtout comme un remède à une maladie de l'âme. Non pas, certes, comme une panacée. Au contraire, je crains qu'elle complique les autres problèmes. (17 mai 1964)

Jean Lesage a déclaré que l'indépendance est la pire menace à notre survie! Quelle bassesse. (2 mai 1966)

L'absurdité la plus effrontée, la plus cynique qui ait jamais été dite ici. (6 mai 1966)

La presse anglaise est unanime à regretter la défaite du parti libéral dans la province de Québec. Ce parti ne favoriserait donc pas tant les Canadiens français? (11 mai 1966)

Hier, terminé la traduction du discours de Donald Gordon. [Refusé au Devoir, Baillargeon travaille au Canadian National.] C'est une charge contre le succès matériel. Il ne s'agit plus là d'un manque de tact seulement, mais indécence. Personne à McGill ne s'en choquera du reste. Les universités qui devraient être les sanctuaires de l'esprit, prodiguent les doctorats honoris causa aux grosses légumes du commerce et de la finance, aux huiles! On mange mieux et surtout on boit plus à leurs tables qu'à celle du pauvre intellectuel canadien! Voilà à quoi je sers ici, à traduire la prose de Donald Gordon! (2 octobre 1965)

Et déjà dans le journal de 1954 !

Qu'est-ce que le bilinguisme? Une langue touristique. S'il est imposé aux habitants d'un pays, il est fatal qu'avec le temps ses habitants parleront la même lanque, celle qui est la plus facile ou la plus utile, et l'anglais, chez nous, est à la fois l'une et l'autre... Le bilinguisme dans le cadre d'une nation déterminée ne peut être que provisoire précédant le choix en toute connaissance de cause entre deux langues données, apprises également, utilisées selon ses besoins, plus ou moins.

La même année, Baillargeon écrit encore:

On sait que le bilinguisme, réellement pratiqué, a toujours eu pour effet de tuer le talent littéraire. II n'existe pas de grands écrivains bilingues. Tout au plus des écrivains apostats qui abandonnent leur idiome naturel pour faire carrière dans un autre.

Ou encore:

Beaucoup de choses devraient être changées chez nous pour assurer la survivance du français. II ne faudrait pas moins changer le milieu où nous vivons, et maintes institutions, et même les mœurs, qui sont étrangères à la culture française et que la langue française est impropre à rendre. 
Vouloir sauvegarder la langue c'est vouloir sauvegarder beaucoup plus que la langue, entre autres choses, tout ce qu'elle seule rend parfaitement. Nous ne pouvons pas vivre dans un milieu étranger, comme des étrangers et parler français... Si Montréal n'a rien de commun avec aucune ville française, le français n'y est plus naturel, ni pratique.

La langue française, approfondie, étudiée avec amour, est un moyen d'aliénation, d'isolement chez nous. On se sépare des siens tout aussi bien en étudiant le français qu'en adoptant l'anglais... Que dis-je: mieux! Car celui qui adopte l'anglais demeure Canadien tandis qu'autrement...

Voilà l'esprit critique de Pierre Baillargeon qui dut souffrir en silence l'infamie de voir vendue l'âme d'un peuple. C'est un Baillargeon qui ne supporte pas la sottise, l'hypocrisie et il est sûr que pour lui, le seul péché était un péché contre l'esprit. Certaines de ses remarques sur le clergé en font un anticlérical certain, mais, comme il aimait le dire, à la façon de Rabelais et non de Voltaire.

Dans le renoncement, trouvant sa volupté? Approfondir cela. De prime abord, me paraissent suspects de mauvaise foi les trouble-fête et leur mépris pour la simple joie de vivre. (5 octobre 1945)

Au déjeuner $X$, je défends les curés et chez les $Y$, je supporte à peine les trois qui s'y trouvent. C'est qu'au fond, je ne change pas tellement de milieu; c'est la sottise qui m'horripile partout. (11 novembre 1945)

Campagnes contre l'immoralité, le blasphème, le cinéma... Voilà les seules campagnes des pauvres. (2 juin 1946)

Notre clergé. II nous organise. II nous nuit aussi. Nous sommes le dernier des peuples, peut-être, à subir le joug d'un autre. Timorés, ignorants, imbéciles, moines, voilà nos acteurs. (20 juillet 1946)

Le clergé est très fort; s'il tombait, il ne resterait rien: il a remplacé la religion. (3 août 1946)

Une des choses les plus cyniques qu'il disait avoir connues, c'était d'entendre lire l'épisode des vendeurs du temple dans une église catholique de la province de Québec. (10 janvier 1961)

Le pape a fustigé la bombe nucléaire que les Américains ont jetée sur Hiroshima. Vingt ans après... (9 août 1965)

Lu le discours du pape à l'occasion de Noël. (...) II fallait au moins ordonner à la chrétienté de passer Noël dans le deuil à cause de tous les enfants tués ou martyrisés au Vietnam depuis si longtemps, sous prétexte de lutter contre le communisme (...) II est immoral de lutter contre une idée avec des armes. (23 décembre 1966)

Après son entrée triomphale à Jérusalem, Christ dit que son royaume n'était pas de ce monde. Combien ont suivi son exemple dans les mêmes circonstances? (1954)

II est coutume et de règle de dire que la survivance française au Canada est l'œuvre de l'Église qui s'est détachée la première de la France et qui a fait chanter un "Te Deum" pour célébrer la victoire des Anglais. (1954)

Mais le journal de Pierre Baillargeon, on le devine bien, parle beaucoup de l'écriture. Constamment, l'écrivain réfléchit sur son œuvre 
à faire, sur les classiques de la littérature française, sur le génie de sa langue, sur les misères de cette langue française en Canada.

Je vieillis, je m'apaise: ce qu'en latin je peux dire en un mot: ematuresco. (1954)

J'ai l'esprit las. Je n'entreprends rien. J'atermoie. Ainsi ferai-je peutêtre jusqu'au jour où je serai convaincu qu'il n'y a pas de conditions favorables et qu'une œuvre s'écrit malgré tout. II me semble encore que je serai lucide, plus en verve, plus libre, plus sûr, plus maître de ma langue, plus moi-même enfin, qu'aujourd'hui. (5 septembre 1945)

Je n'ai plus la force, le courage d'écrire le soir! mes huit heures à la Patrie, mes deux heures en tramway, prennent le meilleur de mon temps. (5 mai 1946)

Je suis un fanatique de la bonne littérature, de la prose française des XVIlè et XVIIlè siècles, de la clarté, de la simplicité, de l'harmonie, de la probité. (17 mai 1946)

Je souffre du mépris avec lequel les éditeurs canadiens traitent les écrivains canadiens.

L'écrivain a bien quelque chose à dire d'abord, mais ce n'est pas ce qu'il dira de meilleur: écrire est sa manière de chercher. Les mots sont des cobayes. (25 août 1946)

Je crois bien que je ferai erreur toutes les fois que je tâcherai de faire autre chose que d'écrire. (4 octobre 1957)

Un seul regret: je n'aurai pas eu le temps d'écrire un livre. C'était ma seule ambition et je $m$ 'y suis préparé toute ma vie à la réaliser un jour. Un autre le fera peut-être mieux. (22 février 1961)

Depuis trente ans, presque tout m'a serré le cœur. (25 janvier 1967) Je ne préconiserai pas mon ouvrage, dit-il. Non pas parce que c'est le mien ni que j'en veuille à mon éditeur. Mais à cause de ce que j'y ai mis: du bon sens, de l'ironie et du style. Le bon sens est décrié par tous les grands hommes du jour. II suffit d'ailleurs de voir comment les gens vivent pour se rendre compte du piètre rôle qu'il joue dans le monde. L'ironie est une source de malentendus. C'est la tournure d'esprit de ceux à qui il n'est pas naturel de crier sur les toits. Enfin, le style, comme le moi, est devenu haïssable: il faut être comme les autres. (14 janvier 1967)

Voilà l'un des derniers témoignages discrets de Baillargeon sur son ceuvre. On voit le tour indirect de ce témoignage du 14 janvier 1967: le classique est effacé. II est surtout généreux, et le dernier témoignage du journal sur l'écriture l'illustre bien:

Le but de l'homme de lettres: donner aux mots la qualité des choses.

Exprimer un peuple. Exprimer l'amour d'un ccur jeune. Faire voir.

Faire apparaître du soleil dans la nuit, en plein jour des étoiles.

Cacher le visible par l'invisible. (27 mai 1967)

On le voit, l'homme intime est noble. Le voilà qui parle de son peuple à la veille de partir pour l'opération à cœur ouvert. Lui qui n'aime pas l'abbé Groulx, image détestable à ses yeux du nationalisme catholique, écrira dans ses dernières pages:

Aucun ministre du gouvernement fédéral n'était présent aux funérailles nationales du chanoine Groulx. (...) Cette absence est un véritable 
camouflet. (27 mai 1967)

C'est sans amertume qu'il écrit:

Mes compatriotes, sans langue, et croyant à une littérature sans style, ne pouvaient s'intéresser beaucoup à mon effort. (28 mai 1967)

L'œuvre de Pierre Baillargeon, c'est celle de l'intelligence en procès. C'est en cela peut-être qu'il est surtout valéryen.

L'ennui, chez Valéry. C'est le fond de son cuvre. II s'en est distrait comme il a pu. Vivre lui pesait. L'illusion de faire quelque chose lui faisait oublier la vanité de tout pour un temps. Son ironie amère. $\mathrm{Sa}$ lucidité triste. Amère et triste ne peuvent signifier là que frustration. Étre à part, avec ce que cela suppose de grand et de limite. Pourtant de la tendresse et nulle fraude. (18 mai 1956)

Contrairement à celui à qui il doit sa recherche de l'intelligence, Baillargeon ne fut pas un désabusé honnête mais un littéraire généreux et presque naï. II dira justement de lui: "J'ai toujours pensé comme si je n'avais pas eu de passion supérieure à celle de penser juste» (journal: pensées écrites en 1954). Et cela Baillargeon le fit passionnément sans passion parce qu'il aimait. II aimait le monde, il aimait la vie, il aimait parler longtemps en buvant un café noir et amer. C'était pour reprendre une expression du journal de 1954 «un solitaire très homme du monde». S'il écrivait sec, il dira aussi: «Écrire sec, comme on dit boire sec.» (1954) Et il s'aimait, oui, de façon exigeante.

C'est envers soi-même qu'on est le plus lâche. II est bien plus facile de se plier au bon plaisir des autres que de s'en tenir impitoyablement au sien. (Journal de 1954)

Paul Toupin, à la fin de son éloge à l'envers s'interroge sur la mort de Pierre Baillargeon:

Quand on m'apprit sa mort, j'étais à Rome, à la recherche de ma santé, ignorant qu'il fut si malade. La nouvelle m'affecta durement. N'avions-nous pas, à distance, mais ensemble, suivi le chemin escarpé et essouflant de la vie? Je me demandais sur qui son regard, avant de ne plus voir, s'était posé; quelle main, en signe d'adieu, avait serré la sienne, et si la dernière parole avait été celle de la souffrance ou de l'apaisement. Je me demandais surtout quel Pierre Baillargeon s'absentait pour toujours. Celui sans doute que je ne connaîtrais jamais ${ }^{26}$.

C'est l'une de ses filles aimées qui lui serrait la main et le Pierre Baillargeon qui partait souffrait, dans un hôpital étatsunien, de ne pas mourir dans la langue qu'il avait défendue et illustrée. Mais Baillargeon avait toujours vécu avec sa mort: il pouvait mourir comme le Bergotte de Proust, lui qui écrivait déjà en 1941:

Mourir entre le rideau et la vitre, lorsque la lune brille au travers de la cage de l'orme, les yeux fixés sur la petite cour pleine de neige bleuâtre.

Voilà bien celui qui écrit en 1954 que «la vue d'un arbre suffit à [l]'enchanter et les ciels du ciel». Celui qui s'absentait avait cru en l'homme de manière tellement humaine: 
La question n'est pas de savoir si l'on croit à Dieu ou à autre chose, mais si l'on croit encore en quelque chose, ne serait-ce qu'à demain. De plus en plus, on agit comme si rien n'avait d'importance, et comme si tout le monde allait disparaître d'un moment à l'autre. Même les plaisirs demandant quelque application sont abandonnés. (6 mars 1957)

Et il ajoute, montrant jusqu'à quel point sa sensibilité est contemporaine, que «ce ne sont pas les différentes croyances qui divisent le plus profondément notre monde, c'est l'espérance tenace et le désespoir " 6 mars 1957). Pour lui, dans sa foi mystérieuse, il croit à un monde en devenir. En 1954, il écrivait de son père mort jeune ce qui convient également pour lui :

J'aimerais pouvoir assurer, et rassurer par là, ceux qui meurent trop tôt, comme lui, que ce qu'ils n'ont pu achever, le fait d'avoir commencé suffit.

Son testament tient dans l'expression du sentiment d'avoir mené la lutte. Dans l'un de ses derniers textes, il écrit:

Le peu que j'ai fait, je l'ai fait malgré tout: malgré le collège, malgré le manque d'intérêt de mes compatriotes pour mes efforts, malgré la fatigue des différents postes subalternes que j'ai dû occuper, malgré ma mauvaise santé en général, malgré mes doutes, malgré le sentiment que j'ai de la vanité de tout ici-bas. (20 avril 1967)

Sa mort sera aussi effacée que son œuvre. "Mourir sans ennuyer son entourage" écrit-il le 19 mai 1967. En prenant congé de la vie, du moins Baillargeon connaissait la gravité de l'intervention chirurgicale qu'il allait subir, il donnera le dernier mot à l'écrivain. Celui qui avait dit dans Commerce que "mourir, c'est fermer une parenthèse ${ }^{27}$ " prendra ses dernières énergies pour écrire ceci, car c'est ainsi que se termine le journal:

Je garde la chambre. Je me médicamente. Je souffre.

Il entend chanter les oiseaux, tandis que le jour se lève.

II regarde par-dessus les toits. De grandes lignes horizontales.

II est presque aérien avant de devenir un pur esprit!

(le 7 juin 1967)

L'homme fait sa révérence à l'écrivain. Il accomplit le chassé-croisé une fois de plus et passe du “je” au “il». Il revient à Hasard et moi écrit à propos du «il » ou de l'écrivain. II meurt en écrivain. II revient à sa jeunesse, il reprend le premier Perrin de Hasard et moi pour compagnon car l'écriture fut son viatique:

Pierre ne peut continuer seul son chemin ${ }^{28}$.

Plutôt que de s'expliquer en vain à Perrin, il [Pierre] se conforme à l'idée de Perrin, et redevient son compagnon ${ }^{29}$.

Puisse Baillargeon devenu pur esprit habiter le corps du Québec qui tremble comme un saule ${ }^{30}$. 
1. Paul Toupin, «Pierre Baillargeon", "Profils littéraires" 2e série, Cahiers de l'Académie canadienne-française, $n^{\circ} 14$, Montréal, 1972, p. 120-130. Ce portrait est repris dans Au commencement était le souvenir, Montréal, Fides, 1973, p. 183-192.

2. Entrevue accordée à Gérald Godin dans le Nouveau Journal: "A la veille de la parution de son théâtre complet, Paul Toupin nous parle: Je suis triste et honteux $", 9$ décembre $1961, p . v$.

3. Lire dans le Choix le texte presque navrant de "Tant de silence». Montréal, HM H, 1969, p. 57.

4. Madeleine Ducrocq-Poirier, «les Méfaits de la subjectivité dans le témoignage littéraire ou les vrais mérites de Pierre Baillargeonm, dans Voix et images du pays VIII. Montréal, PUQ, 1974, p. 127-133. Madame Poirier a fait des études fort intéressantes sur Robert Charbonneau et sur la querelle qui l'opposa aux éditeurs français en 1947.

5. Le personnage principal de Souvenirs pour demain avoue: “Je tiens seulement à écrire un bon livre. Là est ma vie." Montréal, Le Cercle du livre de France, aPoche», 1968, p. 32.

6. «En somme, je n'avais désiré qu'écrire (...) Si chacun a sa vocation, c'était la mienne", affirme à son tour le narrateur de Mon mal vient de plus loin, Montréal, Le Cercle du livre de France, 1969, p. 104-105.

7. Les Medisances de Claude Perrin, Montréal, Parizeau, 1945, p. 66. (Editions du Jour. 1973).

8. Le Choix, p. 40.

9. Le scandale est nécessaire, Montréal, Editions du Jour, 1962, p. 148.

10. Amérique française. novembre 1941, p. 5 .

11. Le Choix, p.17.

12. Le 4 août 1965.

13. Les Médisances, p. 43.

14. Commerce, Montréal, Variétés, 1947, p. 115.

15. Ibid., p. 93.

16. Ibid., p. 114-115.

17. Jean Ménard, "Eloge de Pierre Baillargeon", dans la Vie litteraire au Canada français, Ottawa, Les Presses de l'Université d'Ottawa, 1971, p. 95-102.

18. Dans le Droit, samedi 22 septembre 1973, p. 27: “Moi qui croyais connaître Pierre Baillargeon, j'ai découvert mon ignorance en lisant ce morceau de roi qu'est l'étude de monsieur Toupin. »

19. Les Médisances, p. 120.

20. Ibid., p. 64-65.

21. Ibid., chap. Xvil, «Pourquoi je ne fus pas lu», p. 145.

22. Commerce, p. 183.

23. "Je suis donc un écrivain conservateur, sans aucun goût pour les innovations quelque peu enfantines de mes confrères, tel le remplacement de la virgule par l'espacement des mots, du point virgule par la parenthèse et du tiret par la double parenthèse." Jacques Ferron, Du fond de mon arriere-cuisine, Montréal, Editions du Jour, 1973, p. 237.

24. Baillargeon qui a annoté ici et là la lettre de Ferron a marqué en marge: «Inexact, malheureusement. "

25. Cette lettre est envoyée de Mont-Louis, le 18 janvier 1948, quelques mois avant la parution de la Neige et le feu. Jacques Ferron devait par la suite se porter à la défense de Pierre Baillargeon pris à parti par des lecteurs du Canada scandalisés par le troisième roman du poète-romancier.

26. Voir l'article cité plus haut.

27. Commerce, p. 126.

28. Hasard et moi, p. 27.

29. Ibid., p. 23.

30. Au moment de mettre sous presse, Paul Toupin m'écrit de Monte Alto (Espagne): «Je vous félicite de préparer une thèse sur Pierre Baillargeon. Si j'avais le temps, les moyens et la santé, j'irais à votre soutenance. N'oubliez pas de souligner que le portrait que j'ai fait de lui a bien mal été interprété; on n'a retenu que l'aspect négatif du personnage; pourtant, j'ai bien écrit en toutes lettres qu'il avait du cœur, de l'esprit et du talent... (27 juin 1975). » 